\title{
Nodal Surfaces of Wave Functions of Many-Electron Systems
}

\author{
Evgenii Smolenskii ${ }^{1}$, Anton Ryzhov ${ }^{1}$, Irina Chuvaeva ${ }^{1}$, and Oleg Eliseev ${ }^{1}$ \\ ${ }^{1}$ Zelinsky Institute of Organic Chemistry RAS
}

March 3, 2022

\begin{abstract}
Possible theoretical basis for the hypothesis of prohibition of some electron configurations in atoms and molecules presented by one of the authors earlier is considered. Existence of such prohibited configurations follows from the wave function antisymmetry of atoms and molecules. Our hypothesis is as follows: the configurations, in which the potentials of any two electrons with the same spins are equal, are forbidden. If the hypothesis comes true, it will be possible to find the nodal surfaces of many-electron wave functions for atoms and molecules analytically a priori.
\end{abstract}

\section{Nodal Surfaces of Wave Functions of Many-Electron Systems}

Eugenii A. Smolenskii, 11N. D. Zelinsky Institute of Organic Chemistry Russian Academy of Sciences, Leninsky Prospect, 47, Moscow, Russian Federation, 119991 Anton N. Ryzhov, Irina V. Chuvaeva, Oleg L. Eliseev

Correspondence to: Eugenii A. Smolenskii (E-mail:smolensk@ioc.ac.ru)

\section{Introduction}

The role of antisymmetry requirement of wave functions in the structure of many-electron system configurational space (atoms and molecules, including their excited states [7]) is shown in [1-6]. One of the consequences of this requirement is prohibition for electrons to take some spaces in physical space with zero value of the wave function. These positions of electrons determine some nodal surfaces in configurational space, which divide the space to $N !(N !=1[?] 2[?] 3[?] \ldots[?] N)$ equivalent areas for atoms and molecules with $N$ electrons. The equivalence means that, in case we receive the solution of Schrödinger equation $(\Psi$ function) for one such area, the solution for the rest of them is received simply by permutation of the electron indices [8]. In the same article [8] it is shown that some unknown fundamental physical forces called antisymmetry forces by us must exist. Specifically they forbid to electrons to take the positions mentioned above and characterized usually by some symmetry. In paper [9] the hypothesis was offered that the wave function turns to zero for configurations with equal potentials of any two electrons with the same spins. This hypothesis was proved with calculations for $\mathrm{He}, \mathrm{Li}$ and $\mathrm{Be}$ atoms and for molecules $\mathrm{H}_{2}$ in triplet state and $\mathrm{LiH}[10,11]$. In the present paper same foundation of our hypothesis is given.

\section{Thought experiment}

Let us have a thought experiment. At first, we fixate some configuration for atom or molecule with $N$ electrons, choose two electrons with same spins and give them indices "1" and "2". Let the wave function for this configuration have some value $\Psi 0$ and $\Psi 0>0$ for definiteness (the sign of $\Psi 0$ does not matter). Then the wave function must change sign and turn to $-\Psi 0$ when we change places of electrons indices "1" and "2". We take with fingers or tweezers electrons " 1 " and " 2 " and move them in some arbitrary way changing their places. The other electrons and atom nuclei stay still. In this situation electron "1" runs 
some trajectory and electron "2" runs another trajectory in physical space. The two trajectories in threedimensional physical space can be described by one line in six-dimensional configuration space named by us the interchange trajectory. The spins can be ignored because they coincide.

It turns out that any choice of the interchange trajectory gives $\Psi=0$ at some moment. (For example, we can move electron "1" far away in atomic scale, electron "2" put on its place and then choose some intricate way to move electron "1" to the former place of electron "2" to avoid symmetrical situations). It follows from continuity of the wave functions and the well-known mathematical analysis theorem that proves a continuous function determined on some segment and having opposite signs on its ends turn to zero at least at one point of the segment. We consider the interchange trajectory as such segment. There can be more than one zero points but their number will be odd necessarily. The nodal surfaces of the wave function in configurational space consist of these points.

\section{Original hypothesis}

Let's go back to our hypothesis [9]. Every electron in atom A has some definite value of potential for any configuration of electrons. The value of potential for electron with number $i$ can be expressed by the following formula:

$u_{i}=\frac{N}{r_{A, i}}-\sum_{k \neq i} \frac{1}{r_{\mathrm{ik}}}(1)$

In formula (1) $N$ is the number of electrons in atom A, i.e. the charge of the nucleus, $r_{A, i}$ is the distance between electron with index $i$ and the nucleus, $r_{\mathrm{ik}}$ is the distance from this electron to the electron with index $k$. The summation is being carried for all $\mathrm{k}$ from 1 to $N$ except $i$.

Let's consider function $\Phi=u_{1}-u_{2}$, i.e. the potential difference of electrons "1" and "2" that have the same spins as was pointed before. This function is defined for any point of the interchange trajectory of electrons "1" and "2" and has the same quality as the wave function $\Psi$ itself, i.e. at the ends of the interchange trajectory it has opposite signs according to formula (1). It means that inside the interchange trajectory at least one point where function $\Phi$ turns to zero exists. The meaning of our hypothesis [9] is as follows: for the points with $\Phi=0 \Psi=0$ too, i.e. nodal points of these functions on the interchange trajectory are the same. This means that the set of $\Phi$ nodal surfaces is a subset of the $\Psi$ nodal surfaces set. Function $\Psi$ turns to zero not only for the configurations with $\Phi=0$, i.e. $u_{1}=u_{2}$, but for all the cases $u_{i}=u_{j}$ if spins of electrons " $\mathrm{i}$ " and " $\mathrm{j}$ " are the same. Let's consider the interchange trajectory of electrons " 1 " and "2" with arbitrary coordinates in case when the coordinates of other electrons and nuclei are fixed. Let's the initial distance between the electrons "1" and "2" is decreasing and approaching to zero. Then distances between the zero points of $\Psi$ and $\Phi$ turn to zero too (even if the distances were not the same initially), i.e. these points coincide in the limit. This, of course, is not a proof of our hypothesis but a suggestion giving some hope that it is valid.

\section{Conclusions}

In conclusion, we can point out that in case our hypothesis is proved by calculation for complex atoms and molecules it will give us a possibility to carry quant mechanic calculations on a new high level because then we can determine the boundaries of the above-mentioned $N$ ! equivalent areas analytically a priori . In fact, we suggest a new quant chemistry as it was mentioned in [8].

P.S. It should be admitted that our thought experiment is difficult to carry in physical space for we have to pass through the zero point of wave function. This is forbidden by the forces considered in [8] and responsible for antisymmetry of wave functions. Figuratively speaking, we don't know presently if we have enough energy to overcome the forces of antisimmetry and transfer our electron through this point. This experiment is easier to carry if the coordinates of other electrons are being changed. Of course, we don't know how electrons in atoms and molecules move. Moreover, we can't know this in principle if the uncertainty principle of Heisenberg [12] is valid. By the way, in the same paper [12] Heisenberg for the first time used the term "electron trajectory" and considered several thought experiments with electrons. Electrons don't stay still without doubt. In Stern and Gerlach experiments trajectories of electrons were observed but the electrons 
were free and not connected with atoms nuclei. However, we are sure that science does not stand still and is discovering new and unknown laws of the material world, so the mysterious properties of electrons will be explained together with value and range of action of above-mentioned antisimmetry forces in comparison with other fundamental forces.

\section{Acknowledgments}

The authors are grateful to the Program of Development of Scientific Schools of ZIOC RAS.

Keywords: wave functions, Pauli principle, wave functions antisymmetry, Schrödinger equation.

\section{References and Notes}

1. E.A. Smolenskii,Dokl. Akad. Nauk SSSR .1982, 265 , 160.

2. E.A. Smolenskii, Zhurn. Fiz. Khim . 1988, 62 , 3273.

3. I.V. Stankevich, I.V. Stankevich, Teoret. Mat. Fiz . 1991 , 88, 46.

4. I.V. Stankevich, E.A. Smolenskii, N.S. Zefirov,Dokl. Akad.Nauk SSSR . 1991, $314,213$.

5. I.V. Stankevich, E.A. Smolenskii, N.S. Zefirov, Dokl. Akad.Nauk SSSR . 1991 , $317,413$.

6. E.A. Smolenskii, N.S. Zefirov,Dokl. Akad. Nauk SSSR . 1993 , 329, 40.

7. S.N Maximov, S.A. Shpilkin, E.A. Smolenskii, Phys. Rev. A . 2000 , 61 , 2250.

8. E.A. Smolenskii, Russ. Chem. Bull . 2019 , 68 , 1635-1639.

9. E.A. Smolenskii, Russ. Chem. Bull . 1995 , 44 , 1598.

10. E.A. Smolenskii, S.A. Shpilkin, P.P. Aristov, S.Ya. Itshenko, S.N. Maximoff, J. Chem. Inf. Comp. Sci . $1996,36,402$.

11. N.D Chuvylkin, E.A. Smolenskii, N.S. Zefirov, Russ. Chem. Rev .2005 , 74, 11, 1027-1038.

12. W. Heisenberg, Zeitschrift für Physik . 1927 ,43 , 3, 172. 